\title{
An Electro encephalographic signal Classification in Large Data Set using Deep learning Techniques
}

\author{
${ }^{1}$ Raju Anitha, ${ }^{2}$ Ravi Kumar Tata,${ }^{3}$ V.LakshmaReddy, ${ }^{4}$ M.V.Ramana Moorthy \\ ${ }^{1}$ Associate Professor, Koneru Lakshmaiah Education Foundation, Vaddeswaram, Guntur, AP \\ ${ }^{2}$ Associate professor, Koneru Lakshmiah Educational Foundation Vaddeswaram Guntur, AP \\ ${ }^{3}$ Assistant Professor, Guntur Engineering College, Guntur, AP \\ ${ }^{4}$ Associate Professor Koneru Lakshmiah Educational Foundation Vaddeswaram Guntur, AP
}

ABSTRACT

The measure of openly accessible human characteristics data expanding day by day, but then little is thought about the sorts of derivations or recognizing qualities that could sensibly be drawn from that information utilizing new factual strategies. The brain electrical messages are recorded using Electro-encephalography (EEG) .It is the solitary, most functional tool for diagnose people with suspected epilepsy. If the patients are suffering from epilepsy, the EEG can be worn by finding out what type of epilepsy they have, so doctors can treat them in the most excellent way. The EEG is also a valuable test the neurological conditions that include meningitis, encephalitis, toxic or metabolic encephalo pathies and dementias by assessing the brain functionality. Our techniques could effectively recognize patients taking either anticonvulsant or those taking no meds; just as between the two anticonvulsants. Further, we discussed various ways to deal with be best for various classification techniques[2,3]. A set number of past investigations have taken a gander at neurological marker contrasts utilizing EEG between various anticonvulsant prescriptions so as to estimate the consequence of the medications on psychological execution and

neurological patterns. This paper presents the intending to recognize diverse anticonvulsant medication taken by patients utilizing exclusively neurological movement based on machine learning techniques such as SVM,Randomforest etc.,

Keywords: EEG, Machine Learning Techniques, neurological patterns etc.,

\section{INTRODUCTION}

Electroencephalography (EEG) is one case of an application space with a high potential for sway from AI, as this gadget innovation is progressively being utilized for symptomatic and rehabilitative purposes and as a proxy biomarker for pharmacodynamic demonstrating of drugs. EEG is commonly non-intrusive, generally modest, and takes into consideration direct following of neural populace action with high beat ral goal for target estimations of psychological

capacity and correspondence between cerebrum regions. In spite of the fact that examination into utilizations of AI to EEG has been in progress for 10 years, more work is expected to comprehend its cutoff points in determining data that could advise clinical choices. In particular, sufficient approval informational collections with realized subject or patient attributes are vital so as to evaluate the exactness and ampleness of AI behavior to compact with clinical issues. Up to the present, this need has restricted the size of most exploration studies to few patients. Luckily, the production of enormous clinical data sets with both patient data and comparing physiological information will presently empower further investigation of numerous order issues with clinical importance. By using EEG report the Doctors can gain the information regarding the brain activity. In EEG report alpha waves, Beta waves, Theta waves Delta waves will provide the information concerning the functionality of brain when the human opens eyes or closed.

There are normally two diverse algorithmic approaches to c onsider with its individual merits and demerits those are : deep Learning algorithms or feature-based Algorithms. Even for the experienced persons it is extremely tricky to understand the EEG reports. There are some automated feature exaction methods such as SLN,SCNN,DCNN and EGGNET can be very well and require less expertise knowledge about the data set. In contrast when expert knowledge is available then it requires Feature based approaches such as Support Vector Machine, KSVM and RF. to produce the predictable outcomes[4,7]. In the present work we propose Both classification techniques accuracy is compared to predict the EGG normal and abnormal report. The given figures below reprasets the sample Normal EGG report and abnormal EGG report. 


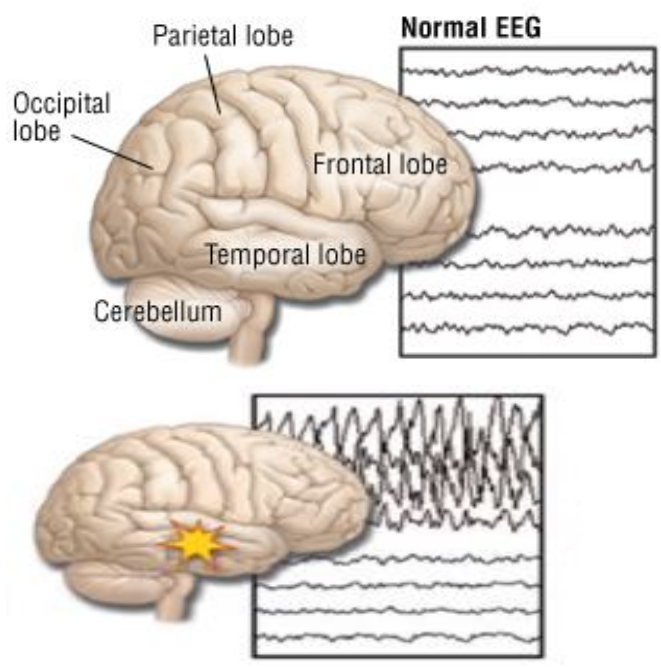

Fig 1: a)Normal EEG Report

b)Abnormal EEG Report

The subsequent figure shows the flow chart representation of the procedure of data preprocessing and classification. At this point we are not involved in data collection of the clinical database in the experiment. Rather, in this paper we found different publicly available resource where we can have the data .For instance github is the publically available repository to gain the data for the experiment. All the preprocessing and analysis was completed by python / Anaconda .

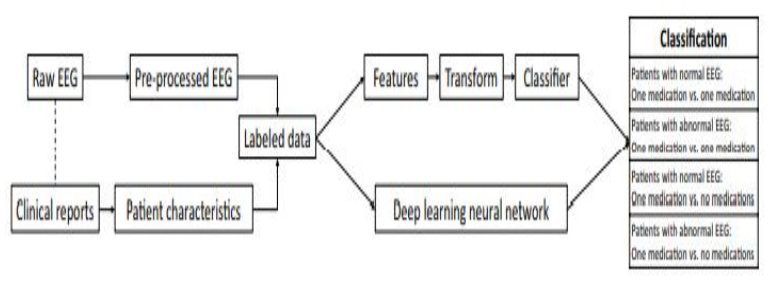

Fig 2: Flow chart for EEG Data preprocessing and Classification

\section{METHODOLOGY}

Usually, the accuracies of featured based classification were comparable with the accurateness of DeepLearning classification. The RF models are identified appreciably different than SVM models .Alternatively the standard deviation of the Deep Learning model is higher than the result of the Feature based models. This was normal given the stochastic idea of the streamlining of DCNN models that produce bigger variety in the cross-approved outcomes as opposed to kSVM's more deterministic methodology. We additionally found that kSVM models yielded lower P-values than comparing DCNN models even with lower cross validation classification exactnesses contrasting subjects and strange EEG .So, we see that the DCNN models had the option to get exactness rates utilizing irregular marks nearer to results acquired from effectively named information. Given the computerized idea of DCNN highlight extraction and as of late demonstrated efficacy in complex informational indexes, DCNN models are relied upon to have the opportunity to find patterns. Here we are discussing the various classification techniques[6].

Feature transformation. As the name implies the Feature transformation method is used to transform the features using max normalization. The primary goal of max normalization is by taking the transform features as input and was functional to the data by finding the mean and maximum value of each feature overall all subjects in the given training data. The both values in training data set and testing data set then subtracted by mean and divided by the maximum value to get the outcome from the training set, transformation scale for each feature is range the values between Zero and 1 in training data and approximately 0 or 1 in testing Data[5].

SVM classifiers.: To conclude optimal dimensionality Reduction method, and hyper-parameters for the linear SVM and nonlinear SVM classifications a grid search was used. This methodology can be effortlessly reached out to nonlinear isolating hyper planes, for example, spiral premise and polynomial capacities[1]. A further bit of leeway of this methodology is that it gives a load to each component showing how much the element adds to the between-class detachment. Correspondingly, uphold vector machine can be likewise utilized as a coordinated element decrease. The grid searches were done on other ten-fold cross validation to refrain the SVM and kSVM models using a validation set extract from the preliminary training set from the testing data set.

Random forest classifier: All calculated and transformed features were utilized in a random forest classifier with hundred estimators and two minimum samples to divide a node, one minimum sample per node[8,10]. On hyper-parameters the grid search was not perform for this technique because we noticed that high training accuracies on all classifications.

Deep convolution neural network classifier: This model take the EEG signals as input and therefore applied a few layers of convolutions and pooling before a dens system classification layer. The ELU is a burly substitute to the frequently used rectified linear unit (ReLU) function because it can create non positive outcomes and reduce slowly until its result equals the parameter value. The same mode is too useful batch normalization with each density and pooling pair. The following figure shows the architecture which is used to show efficient neural network architecture for automated feature extraction.

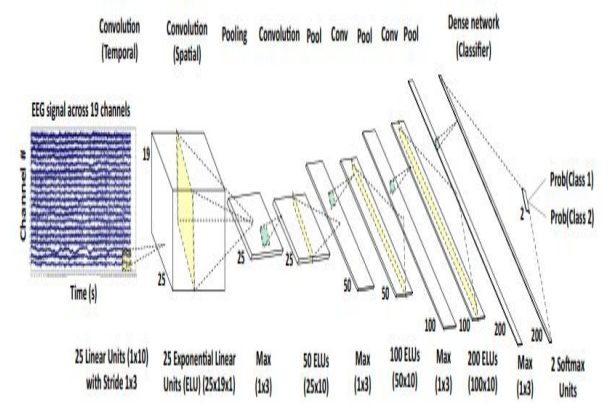

Fig 3: DEEP CONVOLUTION NN ARCHITECTURE 
Raju Anitha et al., International Journal of Emerging Trends in Engineering Research, 8(10), October 2020, $6658-6662$

EEGNet. Classifier: This model takes the input EEG signals and functional more than a few layers of convolutions and pooling before calorific the output for classification with sofmax activation functions to obtain prediction probabilities for each class[11,15]. Once more, the first convolution and pooling pair was different from those utilized in most Deep learning models in light of the structure of EEG information. The two-advance convolution arrangement was likewise stimulated in part by the FBCSP algorithm. However the EEG data is a group of one-dimensional signals where the dimensions are joined .FBCSP inspired two-step sequence as shown before.

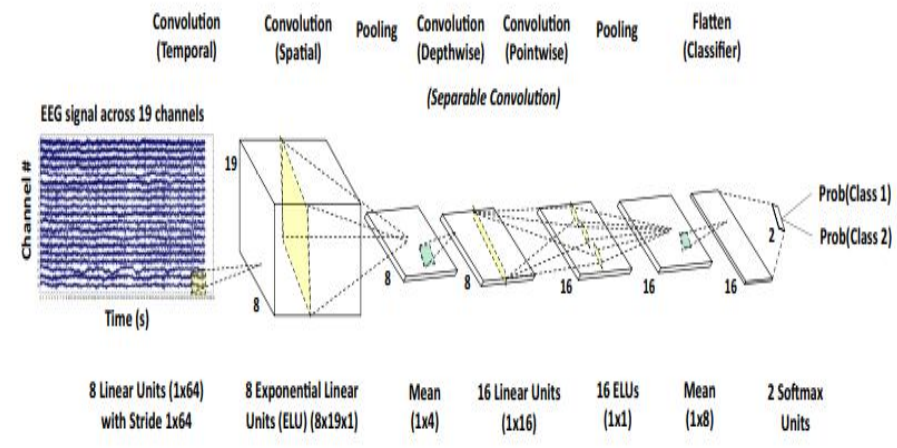

Fig 4: EGG NET ARCHITECTURE

\section{IV.RESULT \&DISCUSSION}

For every double classification, an equivalent number of subjects from every populace was utilized to guarantee equivalent commonness of each condition. $90 \%$ and $10 \%$ of the information were relegated haphazardly to a preparation and testing set, individually. This measure was rehashed multiple times to get classification test results utilizing different subsets of the information for a 10-overlay cross-approved arrangement of results. To gauge the significance of the outcomes, we arbitrarily allocated drug status marks to the preparation information and again acquired ten cross-approved test set classifications dependent on irregular gathering names. We analyzed the test set exactness consequences of the ten models utilizing right names and ten models utilizing haphazardly marked preparing information with a non-parametric Kruskal-Wallis test to decide the names' signifcance. We utilized edges of $\mathrm{P}<$ .01 and $\mathrm{P}<.001$ as two degrees of significance. In the event that the right name test results were like the arbitrary mark results and non-significant P-values were discovered, the names were viewed as non-instructive in the classification task.

Contrasting the correctness's of every classification and strange EEG between techniques, a few strategies were discovered to be significantly different from different techniques. Figure $1 \mathrm{~b}$ shows the consequences of Kruskal-Wallis tests between the 10-overlap cross approvals of techniques with significant results for every classification. We overlook LNN and EEGNet from these examinations since as referenced they didn't find any significant results and see that solitary RF were significantly diferent than SVM results for contrasting subjects and unusual[6]. All things considered, $0.96 \%$ littler than those from the comparing profound learning models.

\begin{tabular}{|l|l|l|l|l|l|l|l|}
\hline \multirow{2}{*}{ Classification method } & \multicolumn{3}{|l|}{ Feature-based (CPU) } & \multicolumn{4}{l|}{ Network-based (GPU) } \\
\cline { 2 - 8 } & SVM & kSVM & RF & LNN & SCNN & DCNN & EEGNet \\
\hline Mean train time (min) & $23.67(0.20)$ & $23.56(0.20)$ & 0.93 & 8.12 & 8.43 & 16.95 & 8.84 \\
\hline
\end{tabular}

Table 1: Mean computation time in minutes for each of seven classification methods

The EEG Net Classifier methodology centers around ascertaining, on a solitary preliminary premise, the pertinence of individual highlights on the subsequent grouping choice. Positive estimations of importance indicate proof supporting the result, while negative estimations of significance indicate proof against the result. We research a few distinct designs of the EEG Netwok classifier by shifting the quantity of channels

\begin{tabular}{|l|l|l|l|l|l|}
\hline Data Set & $\begin{array}{l}\text { Lengt } \\
\mathrm{h}(\mathrm{Sec})\end{array}$ & $\begin{array}{l}\text { Deep } \\
\text { CNN }\end{array}$ & $\begin{array}{l}\text { Sha } \\
\text { llo } \\
\text { wC } \\
\text { NN }\end{array}$ & $\begin{array}{l}\text { EEGNetwork } \\
\text { Classifier-,2) }\end{array}$ & $\begin{array}{l}\text { EEGNe } \\
\text { twork } \\
\text { Classifi } \\
\text { er-8,2 }\end{array}$ \\
\hline $\begin{array}{l}\text { P300-Even } \\
\text { t Related } \\
\text { Potential }\end{array}$ & 1 & $\begin{array}{l}1741 \\
27\end{array}$ & $\begin{array}{l}104 \\
002\end{array}$ & 1066 & 2258 \\
\hline $\begin{array}{l}\text { Error-Rela } \\
\text { ted } \\
\text { Negativity( } \\
\text { ERN) }\end{array}$ & 1.25 & 1699 & 916 & 1082 & 2290 \\
\hline $\begin{array}{l}\text { Movement } \\
\text {-Related } \\
\text { Cortical } \\
\text { Potential( } \\
\text { MRCP) }\end{array}$ & 1.5 & 1757 & 104 & 1098 & 2322 \\
\hline $\begin{array}{l}\text { Sensory } \\
\text { Motor } \\
\text { Rhythm(S } \\
\text { MR) }\end{array}$ & 2 & 1522 & 406 & 796 & 1716 \\
\hline
\end{tabular}

Table 2: Training set parameters For all dataset for all CNN-based models

Here we see the exhibitions of ShallowCNN , FBCSP are fundamentally the same as, recreating past outcomes as announced, while DeepConvNet execution is altogether lower. We additionally observe that EEGNet4-crease inside subject order execution for the Sensory Motor R dataset for each model, found the middle value of on the whole folds and each and every one subjects. Notice the Error bars signify 2 standard blunders of the mean. At this point we see DeepConvNet measurably performed more regrettable than every other model $(\mathrm{p}<0.05)$. ShallowConvNet and EEGNet-8,2 performed comparatively to that of FBCSP 


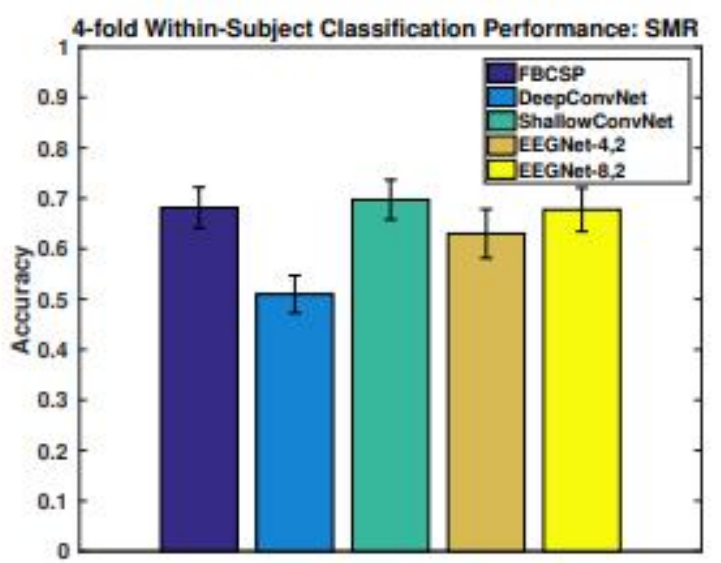

Fig 5: 4-fold classification performance for the SMR dataset for each model

Four-fold subject classification performance for Event Related P300, ERN \&MRCP datasets for each and every model, averaged on the whole folds and all subjects. The specified graph shows the contrast difference of the performance of both the CNN-based reference algorithms (DeepConvolution Neural Neteork and ShallowConvolution Newralnetwork) and traditional approaches with EEGNet-8,2

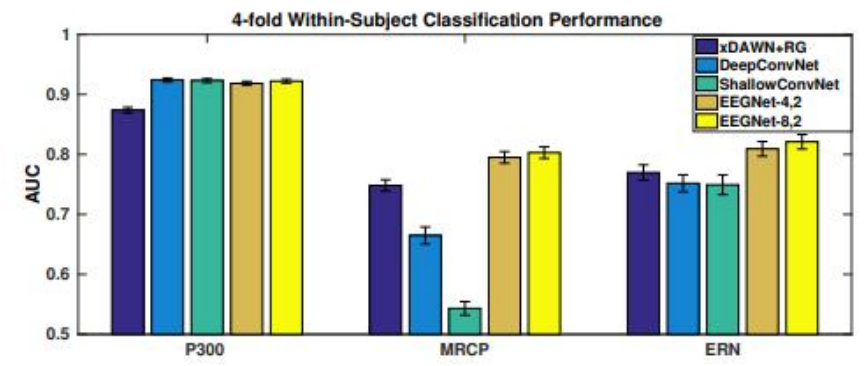

Fig 6: Comparison of all Classification Model for 4 fold within subject

\section{CONCLUSION}

At last, to comprehend the clinical pertinence of these classifications and drug effects on neural movement, examination concerning what attributes of EEG are imperative to these classifications would be instructive. There are a few entrenched strategies accessible to distinguish significant qualities in include based classifications. A portion of these techniques were applied in this investigation alongside cross-approval to distinguish subsets of highlights that performed best in EEG Network Classifier. However we can notice the a significant increase in data dimensionality, therefore requiring either more data or more model regularization or some times both to learn an effective feature representation.

\section{REFERENCES}

[1]Kumar, K. V. V., \& Kishore, P. V. V. (2017). Indian classical dance mudra classification using HOG features and SVM classifier. International Journal of Electrical and Computer Engineering, 7(5), 2537-2546. doi:10.11591/ijece.v7i5.pp2537-2547

[2] Rachapudi, V., Varikuti, V., Anubrolu, J., \& Geethika, C. R. (2017). A comparative analysis of classification algorithms for fetal growth. Journal of Advanced Research in Dynamical and Control Systems, 9, 592-600.

[3] Lakshmi Prasanna, P., \& Rajeswara Rao, D. (2017). Literature survey on text classification: A review. Journal of Advanced Research in Dynamical and Control Systems, 9(Special Issue 12), 2270-2280.

[4] Mitra, A., Si, T., De, A., \& Bhattacharjee, A. K. (2017). A co-segmentation technique using entropy maximization of brain MRI. Journal of Advanced Research in Dynamical and Control Systems, 9(Special Issue 17), 1528-1537.

[5] Wijayanto, I., Hartanto, R., Nugroho, H. A. \& Winduratna, B. Seizure type detection in epileptic EEG signal using empirical mode decomposition and support vector machine. In 2019 International Seminar on Intelligent Technology and Its Applications (ISITIA), 314-319 (2019)

[6] Raghu, S., Sriraam, N., Temel, Y., Rao, S. V. \& Kubben, P. L. EEG based multi-class seizure type classifcation using convolutional neural network and transfer learning. Neural Netw. 124, 202-212.

[7] Golmohammadi, M., Torbati, A. H. N., de Diego, S. T., Obeid, I. \& Picone, J. Automatic analysis of EEGs using big data and hybrid deep learning architectures. Front. Hum. Neurosci. 13, 76.

[8] Bangare, S. L., Pradeepini, G., \& Patil, S. T. (2017). Brain tumor classification using mixed method approach. Paper presented at the 2017 International Conference on Information Communication and Embedded Systems, ICICES 2017, doi:10.1109/ICICES.2017.8070748 .

[9] Vignesh, U., Sivakumar, V., \& Ramana, N. V. (2018). Survey and implementation on classification algorithms with approach on the environment. International Journal of Engineering and Technology(UAE), 7(2.33 Special Issue 33), 438-440.

[10] Zeelan Basha, C. M. A. K., Maruthi Padmaja, T., \& Balaji, G. N. (2018). Automatic X-ray image classification system doi:10.1007/978-981-10-5547-8_5.

[11] Potharaju, S. P., \& Sreedevi, M. (2019). A novel LtR and RtL framework for subset feature selection (reduction) for improving the classification accuracy doi:10.1007/978-981-13-1708-8_20

[12] Rahul, S. (2018). Analysis and presenting the educational techniques in machine and deep learning short communication. International Journal of Engineering and Technology(UAE), 7(4.6 Special Issue 6), 296-298.

[13] Reddy, A. V. N., \& Phanikrishna, C. (2017). Contour tracking based knowledge extraction and object recognition using deep learning neural networks. Paper presented at the Proceedings on 2016 2nd International Conference on Next 
Raju Anitha et al., International Journal of Emerging Trends in Engineering Research, 8(10), October 2020, $6658-6662$

Generation Computing Technologies, NGCT 2016, 352-354. doi:10.1109/NGCT.2016.7877440

[14]Mothe, Saiteja. (2020). "A Model for Assessing the Nature of Car Crashes using Convolutional Neural Networks." International Journal of Emerging Trends in Engineering Research. 859-863. DOI: 10.30534/ijeter/2020/41832020.

[15]Tata, R.K. (2020). "Load balancing analyzer: A recommendation system using machine learning", International Journal of Emerging Trends in Engineering Research. 8(5), pp. 2085-2090. DOI:10.30534/ijeter/2020/99852020 